\title{
Scholarly

\section{Amino Acid Substitution in Par j 2 Recombinant Allergen and Its Effect on IgE Binding Capacity}

\author{
Domenico Nuzzo, Federica Pizzo, Giuseppe Albeggiani, \\ Serafina Sciarrino, and Giovanni Duro
}

Instituto di Biomedicina e Immunologia Molecolare (IBIM), Consiglio Nazionale delle Ricerche (CNR), 90146 Palermo, Italy

Correspondence should be addressed to Giovanni Duro, duro@ibim.cnr.it

Received 31 March 2008; Revised 5 February 2009; Accepted 2 March 2009

Therapeutic attempts to cure allergic diseases reduce symptoms without circumventing the onset of the allergic reaction. Specific immunotherapy (SIT), is the most commonly used treatment. Nevertheless, SIT may account for various adverse events. Therefore, different therapies have been developed in order to treat and prevent allergic reactions. Among these therapies, there is an increased interest in studying recombinant peptides mutated in the IgE binding site. Several studies have shown two major allergens of Parietaria judaica (Pj) named Par 1 and Par j 2, which have been cloned and characterized by us. In our study we have fragmented the Par $\mathrm{j} 2$ protein in order to determine the major epitopes recognized by human IgE and we used site-directed mutagenesis to identify potential amino acid residues involved in IgE binding. The IgE binding activity of the recombinant peptides was tested and the results showed that site-specific mutagenesis at positions K41, T42, T43, and C52 caused a loss of IgE binding. The goal of this work is to synthesize molecules which can induce a protective immune response against $\mathrm{Pj}$. These molecules will be used in immunotherapy in order to create new vaccines for the treatment of Parietaria pollen allergy.

Copyright ( 2009 Domenico Nuzzo et al. This is an open access article distributed under the Creative Commons Attribution License, which permits unrestricted use, distribution, and reproduction in any medium, provided the original work is properly cited.

\section{Introduction}

Immunotherapy is an effective treatment for patients affected by different types of allergic diseases. The mechanism responsible for the success of this treatment is based on Tcell helper capacity of different modulations of the immune response against the allergens. In immunotherapy, allergens are administered in increasing steadily doses and the patient's benefit depends on the allergen concentration used. Nevertheless, the risk of hypersensitivity reactions, mediated by $\operatorname{IgE}$, increases with the amount of injected allergens $[1,2]$.

Therefore the isolation and structural and immunological characterization of the different allergens might be a starting point to build hypoallergenic variants of natural proteins which, used in immunotherapy, could minimize specific immunotherapy (SIT) side effects.

The pollen of Parietaria is a major cause of respiratory allergy in the Mediterranean area, where the most common species are Parietaria judaica $(\mathrm{Pj})$ and Parietaria officinalis. Several studies have found different allergens of $\mathrm{Pj}$ with diverse molecular weights; among these, two, named Par $\mathrm{j} 1$ and Par j 2, have been cloned and well characterized.
Par j 2 is the major allergen, and it is composed of 102 amino acid residues with a molecular weight of approximately $11344 \mathrm{Da}$. Par j 2 is highly allergenic and reacts with $100 \%$ of the sera of the allergic subjects tested. The Par j 2 molecule contains an insert of 622 nucleotides with a correct reading frame of 133 aa and a peptide signal of 31 aa. Following a search on EMBL, we discovered that Par j 2 belongs to a family of proteins named "nonspecific lipid transfer proteins (LTPs)," which are able to transport lipids across the cellular membrane. These proteins possess a preserved secondary structure that forms a motif $\alpha-\alpha-\alpha-\alpha-\beta$ and contain 8 cysteines that form 4 disulphuric bridges that stabilize the three-dimensional structure. [3-8].

The aim of this work is to evaluate the single amino acid contribution to understand IgE/Par $\mathrm{j} 2$ binding by the use of site-specific mutation.

Site-specific mutagenesis can be a valid tool to identify the role of the single amino acid responsible for the allergic response mediated by IgE binding to the allergen. In order to do this, we have studied the functional residues of the protein and made a list of IgE/Par $\mathrm{j} 2$ binding sites.

Among all the available allergenic fragments, the 3855 Par $\mathrm{j} 2$ fragment has been chosen. Its small size makes 


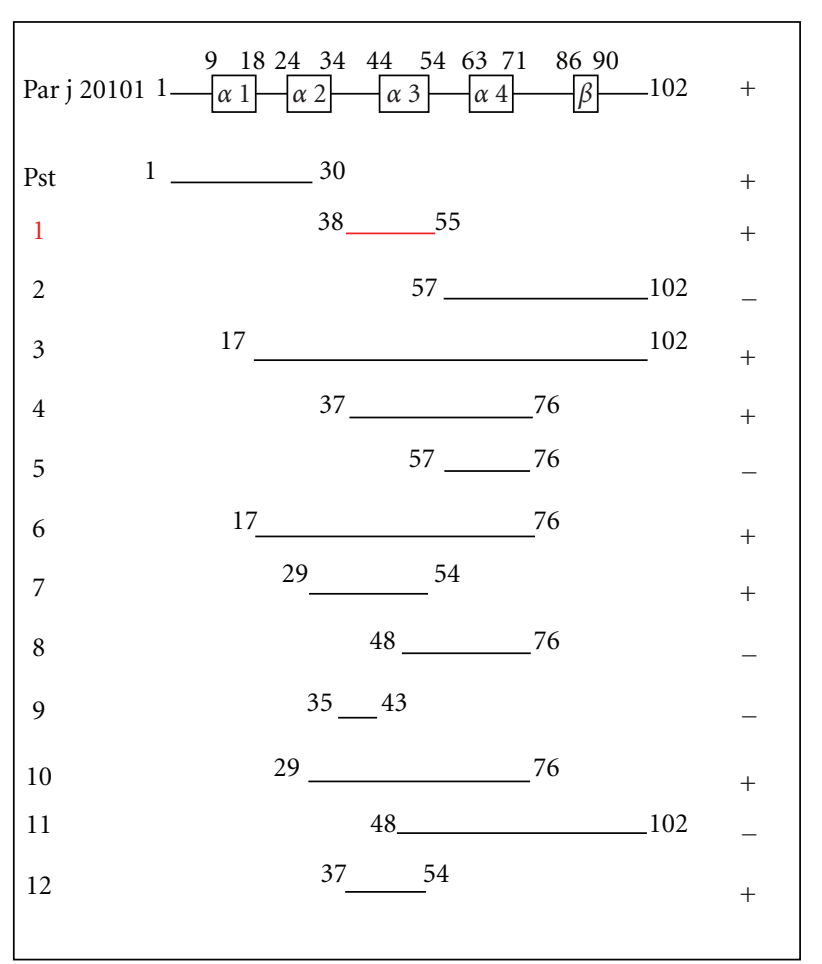

Figure 1: Different Par j 2 fragments of Parietaria judaica $(\mathrm{Pj})$ and their capacity to induce allergic reactions (+/-) (Costa et al.,). Fragment $38-55$ shows positive IgE reaction.

its employment easier. We have made five site-specific mutations and studied the IgE binding activity on the sera of atopic individuals. In our previous studies, these regions have been highlighted as important IgE binding epitopes $[4,6]$ and we have seen that Par $\mathrm{j} 2$ regions 1-30, 30-55, and 77102 were capable of binding specific IgE from sera of allergic patients to PJ (Figure 1), $[4,9]$.

To identify potential amino acid residues directly involved in $\operatorname{IgE}$ binding, site-directed mutagenesis was performed on the region 38-55 [3-6]. The mutants prepared by site-directed mutagenesis were named K41 (2399.66 Da), T42 (2426.73 Da), T43 (2426.73 Da), C50 (2424.69 Da), and C52 $(2424.69 \mathrm{Da})$, where alanine was the amino acid which was replaced. The IgE binding activity of the mutants was tested by Western blot analysis, and the results showed that the site-specific mutations named K41, T42, T43, and C52 caused a loss of IgE binding.

In general, this methodological approach is applicable to other amino acid sequences, regardless of the allergens (pollen, food, insects, etc.). Thus, the use of immunotherapy with compounds produced through site-specific mutagenesis might offer a safer approach to the treatment of allergic disease.

\section{Results}

2.1. Recombinant Peptide Characterization. The immunological activity of the recombinant proteins has been studied through Western blot and analyzed by a 10\% SDS-PAGE

\begin{tabular}{|c|c|}
\hline w.t. & EEVKTTEQKREACKCIVR \\
\hline $\mathrm{K}-41$ & EEVATTEQKREACKCIVR \\
\hline $\mathrm{T}-42$ & EEVKATEQKREACKCIVR \\
\hline $\mathrm{T}-43$ & EEVKTAEQKREACKCIVR \\
\hline C-50 & EEVKTTEQKREAAKCIVR \\
\hline C-52 & EEVKTTEQKREACKAIVR \\
\hline
\end{tabular}

Figure 2: Sequences of wt peptide and site-specific mutations. Through site-specific mutagenesis we replaced the amino acids (coloured in red) with the amino acid alanine.

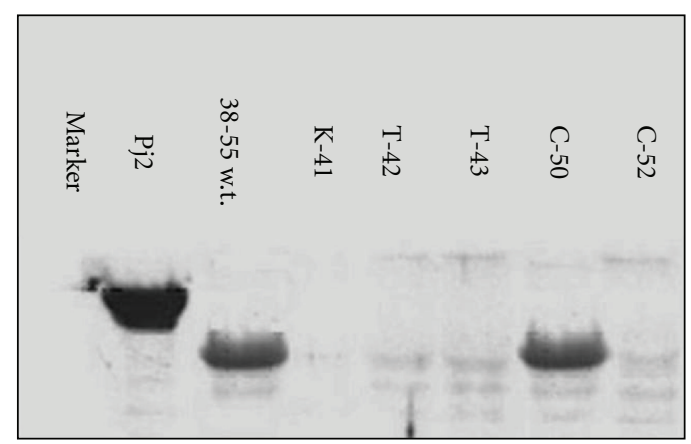

Figure 3: WESTERN bLOT. The picture shows IgE binding between the wt peptide and the mutated recombinants. The filter has been incubated with a pool of sera from subjects allergic to Pj.

gel; the proteins, transferred on a membrane of cellulose, were incubated with a pool of sera $(n=45)$ of patients allergic only to $\mathrm{Pj}$. These patients did not receive any SIT. The five mutations created in the region 38-55 of Par j 2 (K41, T42, T43, C50, and C52) (Figure 2) were inserted in E. coli and purified as a fusion protein using pMALC2 vector. The analysis has shown that clones pMALC2-K41, pMALC2-T42, pMALC2-T43, and pMALC2-C52 lost IgE binding affinity, while the variant pMALC2-C50 maintained the ability to bind to the same antibody, comparable to the wild type and to the positive control, Par j 2 (Figure 3). These results point out that cysteine 50 does not affect IgE binding while other aminoacids are essential for the binding to IgE. All the experiments have been performed using the serum of nonallergic subjects as a negative control.

2.2. Specific IgE Binding. The analysis of recombinant proteins using sera pools from allergic individuals gives an idea of the degree of sensitivity of the population, but it does not give any information about the percentage of single individuals that recognize the allergens.

If we expose each serum to the recombinant peptides, we can detect the degree of individual variability which is the degree of the IgEs binding. Moreover, it is possible 
to find out if the measured value is statistically significant and therefore useful in immunotherapy. The activity of $\operatorname{IgE}$ binding against the five different mutants of Par j 2 was assessed by immunoblot assay using the sera of allergic patients. Figure 4 shows the activity patterns of patients allergic to the pollen of $\mathrm{Pj}$ with each mutant.

These results confirm that each amino acidic substitution introduced by site-specific mutagenesis has important effects on the ability of the recombinant peptides to bind to IgE. The subjects who have specific IgE directed to the 38-55 wild type sequence also recognize the C-50 mutant.

All the experiments have always been performed using the serum of nonallergic subjects as a negative control.

\section{Discussion}

The presence of pollens from flowering plants in the atmosphere can cause allergic reactions in sensitized subjects, who will develop a series of symptoms ranging from simple conjunctivitis or rhinitis to the most severe asthma, deeply affecting their quality of life as well as creating the burden of health expenses.

Our group is devoted to the study and development of immunological therapy against $\mathrm{Pj}$ pollen, the most common form of allergy in the Mediterranean area. Taking in to account that the Mediterranean area is populated by 180 million people, and that around $18 \%$ of the population has one or more forms of allergy and that $\mathrm{Pj}$ pollen affects around $30-50 \%$ of the atopic people, more than 12 million people are subject to this disease. Therefore the social importance and the market interest linked to this specific therapy is clear $[10,11]$. There are several medicines on the market used for alleviating allergy symptoms, but they do not prevent the onset of the allergic reaction. Nowadays, with the use of modern biotechnologies and recombinant DNA, it is possible to isolate and structurally and immunologically characterize many allergens that, used in immunotherapy, could help diminish allergic reactions [2, 12-14]. Allergenic molecules can be modified in such way as to dissociate the useful components (the $\mathrm{T}$ linear epitopes) from the harmful ones (the IgEs B epitopes). Moreover, it has been shown that high doses of allergens modulate $\mathrm{T}$-cell secretive activity inducing IFN- $\gamma$ production rather than IL-4 release [15]. In addition, changes in the molecule's conformational structure that inhibit IgE docking might favor APC activity and therefore T-cell differentiation versus Th0 or Th1 fate $[14,15]$.

The development of safer SIT with allergens that would elicit more moderate side effects would allow the administration of high dose like traditional vaccinations. Also, the route of administration under the tongue or through the nose can improve this therapy [16]. However, there are different possibilities of use, via the administration of single recombinant allergens or a mix of different recombinant allergens. These results represent an important argument for the expansion of new forms of immunotherapy, and several molecules are currently being evaluated in clinical trials. The aim of our group is to produce molecules able to induce a protective immune response against $\mathrm{Pj}$ and in

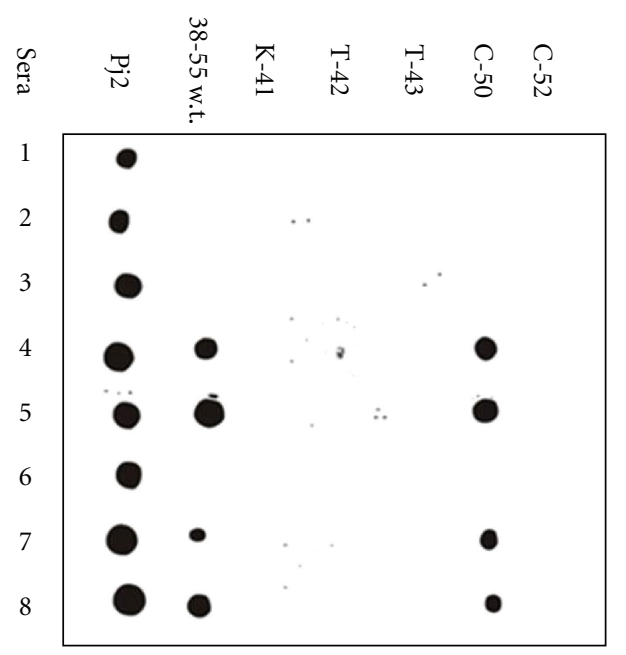

FIgURE 4: DOT blotting assay. Par j 2 recombinant peptides: PAR J 2, 38-55 (wt), K-41, T-42, T-43, C-50, and C-52, are indicated on the top. Filters are numbered from 1 to 8 and indicate different sera of individuals allergic to $\mathrm{Pj}$.

this work we have evaluated, via site-specific mutagenesis, the contribution of single amino acid substitution in the IgE/Par $\mathrm{j} 2$ interaction, the major studied allergens of $\mathrm{Pj}$.

We have predicted the position of Par $\mathrm{j} 2$ functional residues and made a list of those which could influence IgE/Par j 2 interaction. Site-specific mutagenesis can be a valid tool to identify the essential role of single amino acid substitution in immunological response [9, 17]. With this method, the region 38-55 of Par j 2 has been shown to be the most powerful for eliciting allergenic reaction in the serum of atopic individuals. Our results confirm that specific IgEs recognize specific epitopes of Par $\mathrm{j} 2$, and if these epitopes are modified, the IgE affinity declines. These results confirm the validity of the site specific mutations as a tool to make an effective and safer SIT avoiding many anaphylactic reactions. It is known that the inflammatory mediators, responsible for the allergic reactions, are released by mastocytes and basophils when the receptors on their surfaces cross-link with the allergic molecule. Therefore, they must contain at least two sites for IgE binding. On the basis of such knowledge, modified peptides could be used as molecules able to saturate the sites of recognition for the allergens on the mastocyte and basophil surfaces, preventing IgE activation and the subsequent liberation of the inflammatory mediators. A further contribution to the allergic reaction derives from the recognition of the $\mathrm{T}$ epitopes, by allergens. These data together could be used in a therapeutic strategy as an alternative to the one cited above, based on the employment of modified allergens which are unable to bind to IgEs on the surface of the basophils but retain the ability to interact with the $\mathrm{T}$ cells [12].

In conclusion, we have studied the Pj Par $\mathrm{j} 2$ allergen, in order to be used for the development of new usable vaccines in immunotherapy which offers the possibility of a safe approach for the treatment of different allergic illnesses. 
W.T.

AATTC GAG GAG GTG AAG ACG ACG GAG CAG AAG AGG GAG GCC TGC AAG TGC ATA GTC CGC C G CTC CTC CAC TTC TGC TGC CTC GTC TTC TCC CTC CGG ACG TTC ACG TAT CAG GCG GAGCT K41/Ala

AATTC GAG GAG GTG GCC ACG ACG GAG CAG AAG AGG GAG GCC TGC AAG TGC ATA GTC CGC C G CTC CTC CAC $\underline{\text { CGG }}$ TGC TGC CTC GTC TTC TCC CTC CGG ACG TTC ACG TAT CAG GCG GAGCT T42/Ala

AATTC GAG GAG GTG AAG GCC ACG GAG CAG AAG AGG GAG GCC TGC AAG TGC ATA GTC CGC C

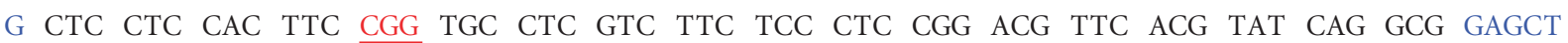
T43/Ala

AATTC GAG GAG GTG AAG ACG GCC GAG CAG AAG AGG GAG GCC TGC AAG TGC ATA GTC CGC C G CTC CTC CAC TTC TGC CGG CTC GTC TTC TCC CTC CGG ACG TTC ACG TAT CAG GCG GAGCT C50/Ala

AATTC GAG GAG GTG AAG ACG ACG GAG CAG AAG AGG GAG GCC GCC AAG TGC ATA GTC CGC C G CTC CTC CAC TTC TGC TGC CTC GTC TTC TCC CTC CGG CGG TTC ACG TAT CAG GCG GAGCT C52/Ala

AATTC GAG GAG GTG AAG ACG ACG GAG CAG AAG AGg GAG GCC TGC AAG GCC ATA GTC CGC C G CTC CTC CAC TTC TGC TGC CTC GTC TTC TCC CTC CGG ACG TTC CGG TAT CAG GCG GAGCT

FIGURE 5: Blue letters indicate the restriction enzyme cloning site, while the red letters indicate the amino acid substitution.

\section{Materials and Methods}

4.1. Cloning in PMALC2 Vector. The wild type sequence was obtained by PCR amplification of the sequence 38-55 of Par $\mathrm{j}$ 2. The cloning sites (EcoRI-XbaI) were added to the PCR primers. One ng of Par j 2.0101 clone was run for 30 cycles under the following conditions: $94^{\circ} \mathrm{C} 30^{\prime \prime}, 52^{\circ} \mathrm{C} 30^{\prime \prime}$, and $72^{\circ} \mathrm{C} 30^{\prime \prime}$. The PCR products were fractioned on $1.8 \%$ agarose gel, and after several steps of purification, digested with EcoRI and XbaI restriction enzymes. The fragment was cloned in the EcoRI-XbaI sites of the pMALC2 vector (BioLabs, UK). The clone was sequenced and the open reading frames confirmed $[8]$.

4.2. Site-Specific Mutagenesis. Mutant oligonucleotides were used to generate a panel of amino acid mutation in the 3855 region. The mutations were carried out at the following positions: K41, T42, T43, C50, and C52, replacing the single amino acid with alanine. The oligonucleotides were synthesized by Pharmacia Biotech, USA. The oligonucleotides were added to another additional cloning site (EcoRI-XbaI). The corresponding oligonucleotides were annealed at $80^{\circ} \mathrm{C}$ for $2^{\prime}$ and slowly cooled at room temperature. The fragments were cloned in the EcoRI-XbaI sites of pMALC2 vector (BioLabs, $\mathrm{UK})$. All the clones were sequenced and the open reading frames were confirmed (see Figure 5).

4.3. Preparation of the Recombinant Proteins. The recombinant clones were grown at $37^{\circ} \mathrm{C}$ to a density of $0.5-0.6 \mathrm{OD}_{600}$ in $\mathrm{LB}$ broth with the appropriate antibiotic and induced for 2 hours with $0.3 \mathrm{mM}$ isopropylthio- $\beta$-D-galactoside. The cells were harvested by centrifugation at $4000 \mathrm{~g}$ for 20 minutes, and the pellet was then dissolved in PBS
(10 mM sodium phosphate, $\mathrm{pH} 7.2 ; 200 \mathrm{mM} \mathrm{NaCl} ; 1 \mathrm{mM}$ EDTA, $1 \mathrm{mM} \mathrm{NaN}_{3}$ ) and lyzed by sonication with the Heat System Ultrasonic, W-385. The cell debris was removed by centrifugation at $9000 \mathrm{X}$ g for 30 minutes. The recombinant proteins were purified by affinity chromatography on an amylase resin column (BioLabs, UK). The concentration of the recombinant proteins was determined by densitometric analysis of SDS-PAGE stained with Comassie brilliant blue and compared to a standard protein.

4.4. Immunoblot. A $2 \mu \mathrm{g}$ amount of each recombinant protein was fractionated on 10\% SDS-PAGE and electroblotted onto PVDF membrane (Immobilon P Millipore, USA). After blotting, membranes were incubated for 3 hours with blocking buffer (PBS supplemented with 3\% BSA, $0,5 \%$ Tween-20, and $0,02 \% \mathrm{NaN}_{3}$ ) and washed three times with PBS containing $0,1 \%$ Tween-20. The filters were then incubated overnight with sera of allergic patients. After washing, the filters were incubated for 45 minutes with horseradish peroxidase HRP-conjugated rabbit antihuman IgE (Sigma, USA). The final reaction was developed with an ECL detection system (Amersham, USA).

\section{References}

[1] A. K. Abbas, A. H. Lichtman, and J. S. Pober, Cellular and Molecular Immunology, W.B. Saunders, Philadelphia, Pa, USA, 2000.

[2] W. Burks, G. Bannon, and S. B. Lehrer, "Classic specific immunotherapy and new perspectives in specific immunotherapy for food allergy," Allergy, vol. 56, no. 67, pp. 121$124,2001$.

[3] A. Amoresano, P. Pucci, G. Duro, et al., "Assignment of disulphide bridges in Par j 2.0101, a major allergen of 
Parietaria judaica pollen,” Biological Chemistry, vol. 384, no. 8, pp. 1165-1172, 2003.

[4] P. Colombo, D. Kennedy, T. Ramsdale, et al., "Identification of an immunodominant IgE epitope of the Parietaria judaica major allergen," The Journal of Immunology, vol. 160, no. 6, pp. 2780-2785, 1998.

[5] M. A. Costa, P. Colombo, V. Izzo, et al., "cDNA cloning, expression and primary structure of $\operatorname{Par} j \mathrm{I}$ a major allergen of Parietaria judaica pollen," FEBS Letters, vol. 341, no. 2-3, pp. 182-186, 1994.

[6] M. A. Costa, G. Duro, V. Izzo, et al., "The IgE-binding epitopes of rPar j 2, a major allergen of Parietaria judaica pollen, are heterogeneously recognized among allergic subjects," Allergy, vol. 55, no. 3, pp. 246-250, 2000.

[7] G. Duro, P. Colombo, M. A. Costa, et al., "Isolation and characterization of two cDNA clones coding for isoforms of the Parietaria judaica major allergen Par j 1.0101," International Archives of Allergy and Immunology, vol. 112, no. 4, pp. 348355, 1997.

[8] G. Duro, P. Colombo, M. A. Costa, et al., "cDNA cloning, sequence analysis and allergological characterization of Par $\mathrm{j}$ 2.0101, a new major allergen of the Parietaria judaica pollen," FEBS Letters, vol. 399, no. 3, pp. 295-298, 1996.

[9] E. Pace, G. Duro, S. La Grutta, et al., "Hypoallergenic fragment of Par $\mathrm{j} 2$ increases functional expression of Toll-like receptors in atopic children," Allergy, vol. 61, no. 12, pp. 1459-1466, 2006.

[10] M. Masullo, S. Mariotta, L. Torrelli, E. Graziani, S. Anticoli, and F. Mannino, "Respiratory allergy to Parietaria pollen in 348 subjects," Allergologia et Immunopathologia, vol. 24, no. 1, pp. 3-6, 1996.

[11] B. Wuthrich, "Epidemiology of allergic diseases: are they really on the increase?" International Archives of Allergy and Immunology, vol. 90, pp. 3-10, 1989.

[12] F. Ferreira, P. Briza, D. Inführ, et al., "Modified recombinant allergens for safer immunotherapy," Inflammation \& Allergy Drug Targets, vol. 5, no. 1, pp. 5-14, 2006.

[13] J. N. Francis and M. Larché, "Peptide-based vaccination: where do we stand?" Current Opinion in Allergy and Clinical Immunology, vol. 5, no. 6, pp. 537-543, 2005.

[14] M. Larché, C. A. Akadis, and R. Valenta, "Immunological mechanisms of allergen-specific immunotherapy," Nature Reviews Immunology, vol. 6, no. 10, pp. 716-771, 2006.

[15] J. Rolland and R. O'Hehir, "Immunotherapy of allergy: anergy, deletion, and immune deviation," Current Opinion in Immunology, vol. 10, no. 6, pp. 640-645, 1998.

[16] K. Takabayashi, L. Libet, D. Chisholm, J. Zubeldia, and A. A. Horner, "Intranasal immunotherapy is more effective than intradermal immunotherapy for the induction of airway allergen tolerance in Th2-sensitized mice," The Journal of Immunology, vol. 170, no. 7, pp. 3898-3905, 2003.

[17] Y. Ma, G. Gadermaier, B. Bohle, et al., "Mutational analysis of amino acid positions crucial for IgE-binding epitopes of the major apple (Malus domestica) allergen, Mal d 1," International Archives of Allergy and Immunology, vol. 139, no. 1, pp. 53-62, 2005. 